\title{
Trajectory Design with Hybrid Low-Thrust Propulsion System
}

\author{
Giovanni Mengali* and Alessandro A. Quarta ${ }^{\dagger}$ \\ University of Pisa, 56122 Pisa, Italy \\ DOI: $10.2514 / 1.22433$
}

\begin{abstract}
A novel mission concept based on a hybrid low-thrust propulsion system is proposed and discussed. A solar electric propulsion thruster is coupled with an auxiliary system providing an inverse square radial thrust. In this way the spacecraft is virtually subjected to a reduced gravitational solar force. The primary purpose of this paper is to quantify the impact of the reduced solar force on the propellant consumption for an interplanetary mission. To this end the steering law that minimizes the propellant consumption for a circle-to-circle rendezvous problem is found using an indirect approach. The hybrid system is compared with a conventional solar electric thruster in terms of payload mass fraction deliverable for a given mission. A tradeoff between payload size and trip time is established.
\end{abstract}

\begin{tabular}{lll} 
& & \multicolumn{1}{c}{ Nomenclature } \\
$A$ & $=$ & sail area \\
$f_{m}$ & $=$ & auxiliary function [see Eq. (26)] \\
$H$ & $=$ & Hamiltonian \\
$H^{\prime}$ & $=$ & Hamiltonian that explicitly depends on the control \\
$J$ & $=$ & pector \\
$m$ & $=$ & mass \\
$r$ & $=$ & sun-spacecraft distance \\
$T$ & $=$ & thrust \\
$t$ & $=$ & time \\
$u$ & $=$ & radial velocity \\
$\boldsymbol{u}$ & $=$ & control vector \\
$v$ & $=$ & circumferential velocity \\
$v_{c}$ & $=$ & circular velocity \\
$v_{e}$ & $=$ & electric thruster exhaust velocity \\
$v_{\mathrm{esc}}$ & $=$ & escape velocity \\
$x$ & $=$ & ratio between the orbital radii \\
$\alpha$ & $=$ & electric thruster direction angle \\
$\beta_{\sigma}$ & $=$ & dimensionless sail loading \\
$\beta_{\sigma}$ & $=$ & weighted dimensionless sail loading \\
$\Delta V$ & $=$ & total variation of velocity \\
$\eta$ & $=$ & ratio of the auxiliary system thrust force to the solar \\
$\theta$ & $=$ & gravitational force \\
$\lambda$ & $=$ & polar angle \\
$\mu$ & $=$ & gravint variable \\
$\sigma$ & $=$ & sail loading \\
$\tau$ & $=$ & electric thruster switching parameter \\
$\tau_{a}$ & $=$ & solar sail switching parameter \\
$\Psi$ & $=$ & sail optical efficiency \\
& &
\end{tabular}

Subscripts

$\begin{array}{lll}a & = & \text { auxiliary } \\ e & = & \text { solar electric propulsion system } \\ f & = & \text { final } \\ \max & = & \text { maximum } \\ \text { pay } & = & \text { payload } \\ \text { prop } & = & \text { propellant }\end{array}$

Received 13 January 2006; revision received 11 May 2006; accepted for publication 12 May 2006. Copyright (C) 2006 by Giovanni Mengali and Alessandro A. Quarta. Published by the American Institute of Aeronautics and Astronautics, Inc., with permission. Copies of this paper may be made for personal or internal use, on condition that the copier pay the $\$ 10.00$ per-copy fee to the Copyright Clearance Center, Inc., 222 Rosewood Drive, Danvers, MA 01923; include the code 0731-5090/07 \$10.00 in correspondence with the CCC.

*Associate Professor, Department of Aerospace Engineering; g.mengali@ ing.unipi.it. Member AIAA.

'Research Assistant, Department of Aerospace Engineering; a.quarta@ ing.unipi.it. Member AIAA.

$$
\begin{array}{lll}
\text { sail } & = & \text { sail assembly } \\
0 & = & \text { initial } \\
* & = & \text { critical } \\
\odot & = & \text { sun } \\
\circ & = & \text { Venus } \\
+ & = & \text { Mars }
\end{array}
$$

Superscripts

. $=$ time derivative

\section{Introduction}

$\mathbf{N}$ EW mission concepts are increasingly considering the use of low-thrust propulsion for efficient navigation in deep space. The employment of low-thrust propulsion technologies is especially interesting for those missions requiring large changes in orbital energy $[1,2]$. The closely related problem of trajectory optimization is also highly attractive from a theoretical viewpoint due to the inherent difficulties in finding optimal trajectories characterized by continuous thrust over long time periods.

Several deep-space missions have been identified that can be performed using solar electric propulsion (SEP) so as to significantly reduce the total mission costs. These missions include Venus surface sample return, Saturn ring observer, Titan explorer, Neptune orbiter, and various Mars sample return options [3]. Other low-thrust propulsion systems, such as solar sails and minimagnetospheric plasma propulsion, have been considered for primary propulsion systems in interplanetary missions, for example, see [4,5].

The choice among the different propulsion concepts is driven by various mission requirements and constraints, among which the total mission cost has a central role. The purpose of this paper is to investigate the potentialities introduced by integrating two different low-thrust propulsion concepts and to highlight the tradeoff between costs and flight time.

The starting point of our analysis is that heliocentric transfers could benefit from a reduced gravitational solar force acting on the spacecraft. In a recent paper McInnes [6] introduced the concept of generalized orbits, that is, orbits obtained through a modulated inverse square radial thrust. In essence, the idea consists of studying the motion of a spacecraft under the effect of a gravitational force whose magnitude is reduced with respect to the solar gravity. The size of this reduction, quantifiable through a dimensionless parameter that will be referred to as $\eta$, is, to some extent, variable and capable of being modulated as a function of time. With such a model different families of orbits can be generated, and the problem of calculating open escape orbits and transfers between circular coplanar orbits can be approached.

The maximum orbit radius attainable using an inverse square radial thrust is $r=r_{0} /\left(1-\eta_{\max }\right)$ [6], where $\eta_{\max }$ is the maximum value of $\eta$. There are at least two types of low-thrust propulsion, 
namely, solar sail and minimagnetospheric plasma propulsion, that can generate thrust-induced forces varying as the inverse square of the heliocentric distance. For both propulsion types, however, the current and near term technology can guarantee only small $\eta$ values. Assuming for instance $\eta_{\max }=0.5$ (which is well beyond the current technology), the maximum orbit radius attainable using an inverse square radial thrust is only $2 r_{0}$. This result substantially limits the practical applicability of such a transfer technique, the more so for escape orbits.

For these reasons we investigate a hybrid low-thrust propulsion (HLTP) system in which a system capable of providing an inverse square radial thrust is coupled with a SEP system. The idea of combining different low-thrust propulsion systems has been introduced by Leipold and Götz [7]. In their preliminary study [7], the HLTP concept was used to assess the feasibility of the idea and roughly estimate the performance enhancements, in terms of mission time, of a solar sail combined with a SEP system with respect to a pure solar sail.

The primary purpose of this paper is to systematically quantify the impact of the control parameter $\eta$ on the propellant consumption for a transfer circle-to-circle problem. To make a meaningful comparison between a HLTP and a conventional SEP system, these two options are compared in terms of payload mass fraction deliverable for a given mission. Also, the tradeoff between payload size and trip time is established.

\section{Quasi-Hohmann Transfer}

To better appreciate the potentialities of a hybrid propulsion system, we begin our discussion by considering a biimpulsive transfer between circular coplanar orbits for a spacecraft with an auxiliary inverse square radial thrust, capable of providing a constant value $\eta \equiv \eta_{\max }<1$. In particular, we investigate the mission performance, in terms of required $\Delta V$ and transfer time $t_{f}$, for an elliptical transfer orbit tangent to both the initial and final circular orbits with radii $r_{0}$ and $r_{f}$, respectively. This analysis extends the familiar Hohmann results to a quasi-Hohmann transfer, characterized by a spacecraft subject to a gravitational attraction equal to $\left(1-\eta_{\max }\right)$ times that it would experience without the presence of the auxiliary propulsive system.

Introducing the ratio $x \triangleq r_{f} / r_{0}$ between the two orbital radii, the energy equation yields

$$
\frac{\Delta V}{\sqrt{\mu_{\odot} / r_{0}}}=\operatorname{sgn}(x-1)\left[(x-1) \sqrt{\frac{2\left(1-\eta_{\max }\right)}{x(x+1)}}+\frac{1}{\sqrt{x}}-1\right]
$$

where $\operatorname{sgn}(\cdot)$ is the signum function. The flight time divided by the parking orbit period is equal to one-half the transfer orbit period and is given by [6]

$$
\frac{t_{f}}{2 \pi \sqrt{r_{0}^{3} / \mu_{\odot}}}=\sqrt{\frac{(1+x)^{3}}{32\left(1-\eta_{\max }\right)}}
$$

Figure 1 shows that the $\Delta V$ saving with respect to a case without an auxiliary propulsion system $\left(\eta_{\max }=0\right)$ is an increasing function of $\eta_{\max }$. Correspondingly, the mission time tends to increase as $\eta_{\max }$ grows large. Figure 2 specializes the preceding results for an EarthVenus and Earth-Mars rendezvous mission.

\section{Problem Statement}

Assume that the spacecraft has a HLTP system, constituted by a primary SEP system and an auxiliary system capable of generating a modulated purely radial thrust (that is, parallel to the sun-spacecraft direction) and variable according to the inverse square law with the distance $r$ from the sun. At a generic time instant the thrust by the SEP and the auxiliary system are referred to as $T_{e}$ and $T_{a}$, respectively. While $T_{e}$ is constant, the auxiliary system produces a thrust in the form
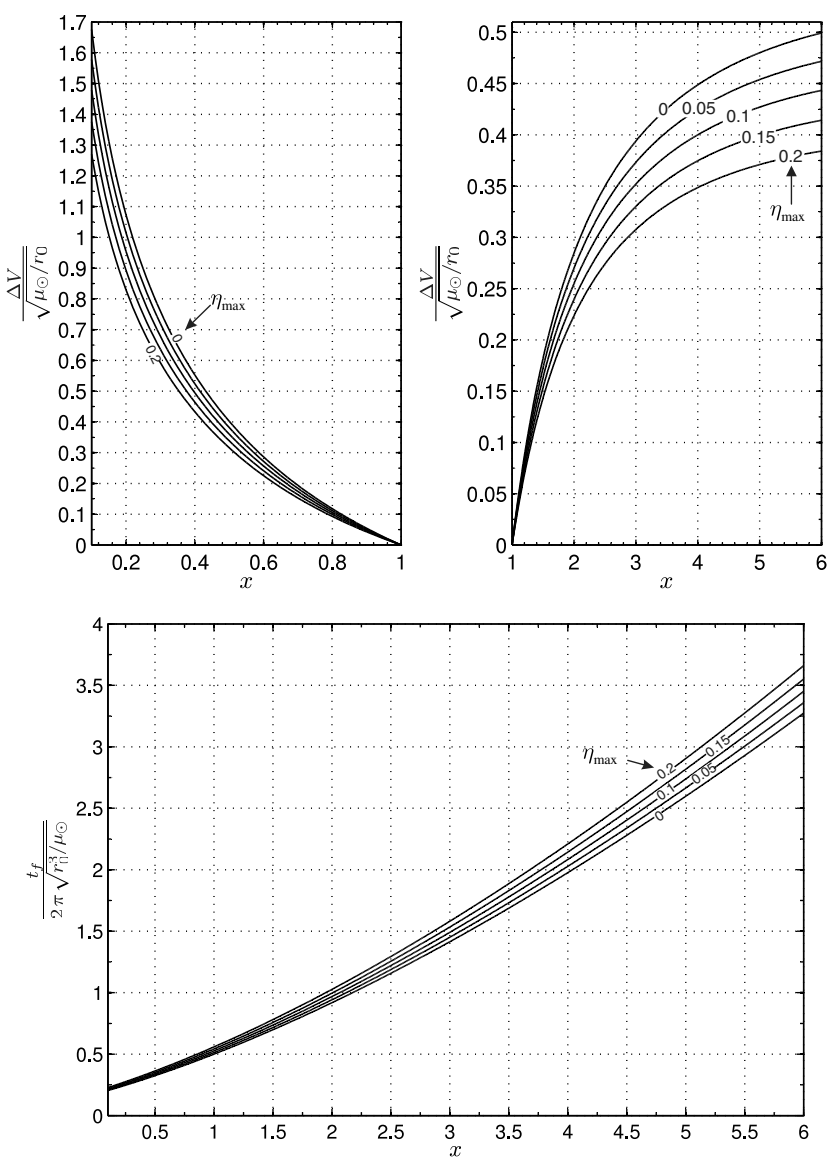

Fig. $1 \Delta V$ and mission time required for a quasi-Hohmann transfer.
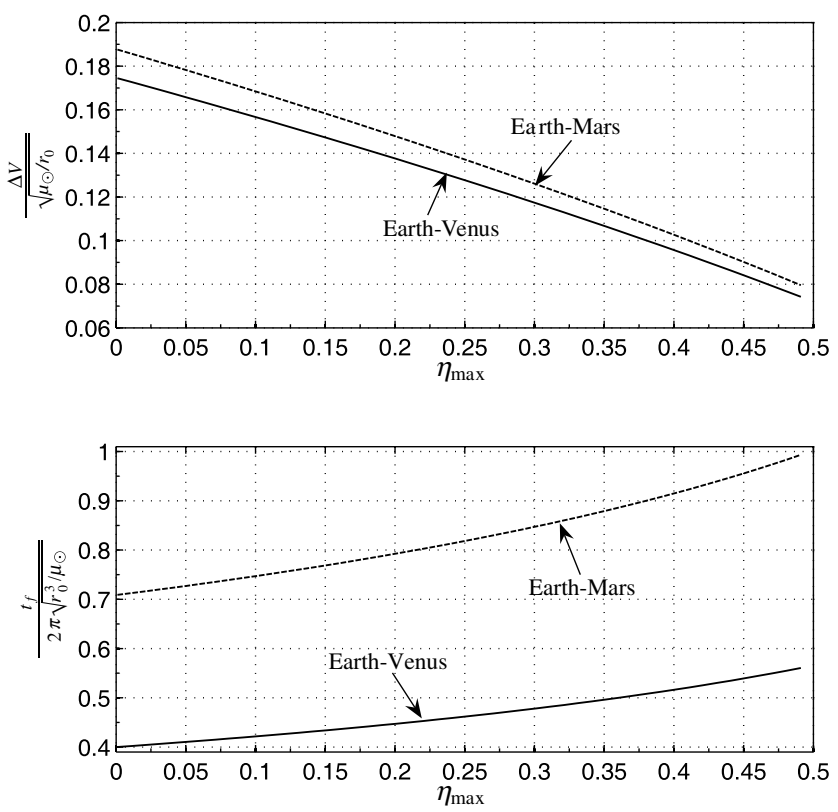

Fig. $2 \Delta V$ variation and flight time for a quasi-Hohmann transfer towards Venus and Mars.

$$
T_{a}=m \eta \frac{\mu_{\odot}}{r^{2}}
$$

where $m$ is the (time variable) spacecraft mass, and $\eta \in\left[0, \eta_{\max }\right]$ is the ratio of the auxiliary thrust force to the solar gravitational force. The main effect of the auxiliary system, which, by assumption, has a null propellant consumption, is to reduce the gravitational acceleration by a factor of $(1-\eta)$. 
The heliocentric equations of motion for a spacecraft in a polar inertial frame $\mathcal{T}_{\odot}(r, \theta)$ are

$$
\begin{gathered}
\dot{r}=u \\
\dot{\theta}=\frac{v}{r} \\
\dot{u}=\frac{v^{2}}{r}-(1-\eta) \frac{\mu_{\odot}}{r^{2}}+\frac{\tau T_{e}}{m} \cos \alpha \\
\dot{v}=-\frac{u v}{r}+\frac{\tau T_{e}}{m} \sin \alpha \\
\dot{m}=-\frac{\tau T_{e}}{v_{e}}
\end{gathered}
$$

where $\theta$ is measured counterclockwise from some reference position, $\tau=(0,1)$ is the electric thruster switching parameter, and $\alpha \in$ $[0,2 \pi]$ is the electric thruster direction angle (in the orbital plane) measured counterclockwise from the radial direction. Clearly, the system of differential equations (4-8) is similar to that describing the two-dimensional motion of a spacecraft equipped with a SEP system, and widely studied in the literature (see, e.g., [8,9]), with the addition of the further control parameter $\eta$ that accounts for the presence of the auxiliary propulsive system.

Our aim is to evaluate the impact of the auxiliary system on the spacecraft trajectories for a rendezvous mission between heliocentric, circular, and coplanar orbits of given radii $r_{0}$ (starting orbit) and $r_{f}$ (target orbit). To establish a meaningful comparison, we investigate optimal trajectories in terms of minimum propellant consumption necessary to accomplish the mission. From a mathematical standpoint the problem is that of finding the optimal control law $\boldsymbol{u}(t)$ (where $t \in\left[t_{0}, t_{f}\right]$ ) that minimizes the propellant mass necessary to transfer the spacecraft from the initial to the final orbit. Equivalently, the performance index

$$
J=m_{f}
$$

must be maximized, where $m_{f}$ is the spacecraft mass at the final time.

\section{Optimal Steering Law}

From Eqs. (4-8), the Hamiltonian associated with the problem is

$$
\begin{aligned}
H & =\lambda_{r} u+\lambda_{\theta} \frac{v}{r}+\lambda_{u}\left[\frac{v^{2}}{r}-(1-\eta) \frac{\mu_{\odot}}{r^{2}}+\frac{\tau T_{e}}{m} \cos \alpha\right] \\
& +\lambda_{v}\left(-\frac{u v}{r}+\frac{\tau T_{e}}{m} \sin \alpha\right)-\lambda_{m} \frac{\tau T_{e}}{v_{e}}
\end{aligned}
$$

where $\lambda_{r}, \lambda_{\theta}, \lambda_{u}, \lambda_{v}$, and $\lambda_{m}$ are the adjoint variables associated with the state variables $r, \theta, u, v$, and $m$, respectively. The time derivatives of the adjoint variables are provided by the Euler-Lagrange equations

$$
\begin{gathered}
\dot{\lambda}_{\theta}=0 \\
\dot{\lambda}_{r}=\frac{\lambda_{\theta} v}{r^{2}}+\lambda_{u}\left[\frac{v^{2}}{r^{2}}-\frac{2(1-\eta) \mu_{\odot}}{r^{3}}\right]-\lambda_{v} \frac{u v}{r^{2}} \\
\dot{\lambda}_{u}=-\lambda_{r}+\lambda_{v} \frac{v}{r} \\
\dot{\lambda}_{v}=-\frac{\lambda_{\theta}}{r}-2 \frac{\lambda_{u} v}{r}+\frac{\lambda_{v} u}{r}
\end{gathered}
$$

$$
\dot{\lambda}_{m}=\frac{\tau T_{e}}{m^{2}}\left(\lambda_{u} \cos \alpha+\lambda_{v} \sin \alpha\right)
$$

Assuming that the final time is left free, the boundary conditions for the differential problem described by the equations of motion (4-8) and the Euler-Lagrange Eqs. (12-15) are initial boundary conditions

$$
\begin{aligned}
& r\left(t_{0}\right)=r_{0}, \quad \theta\left(t_{0}\right)=u\left(t_{0}\right)=0 \\
& v\left(t_{0}\right)=\sqrt{\mu_{\odot} / r_{0}}, \quad m\left(t_{0}\right)=m_{0}
\end{aligned}
$$

and final boundary conditions

$$
\begin{aligned}
& r\left(t_{f}\right)=r_{f}, \quad \lambda_{\theta}\left(t_{f}\right)=u\left(t_{f}\right)=0 \\
& v\left(t_{f}\right)=\sqrt{\mu_{\odot} / r_{f}}, \quad \lambda_{m}\left(t_{f}\right)=1
\end{aligned}
$$

The preceding conditions are representative of a spacecraft leaving the sphere of influence with zero hyperbolic excess velocity. From Eqs. (11) and (18) it follows that $\lambda_{\theta}=0$.

Invoking the Pontryagin's maximum principle, the optimal control law is obtained by maximizing that portion $H^{\prime}$ of the Hamiltonian that explicitly depends on the control vector $\boldsymbol{u} \triangleq$ $[\eta, \tau, \alpha]$

$$
H^{\prime} \triangleq \lambda_{u}\left[(\eta-1) \frac{\mu_{\odot}}{r^{2}}+\frac{\tau T_{e}}{m} \cos \alpha\right]+\lambda_{v} \frac{\tau T_{e}}{m} \sin \alpha-\lambda_{m} \frac{\tau T_{e}}{v_{e}}
$$

As far as $\alpha$ is concerned, the optimal control law is well known [8,9] and is given by

$$
\cos \alpha=\frac{\lambda_{u}}{\sqrt{\lambda_{u}^{2}+\lambda_{v}^{2}}} ; \quad \sin \alpha=\frac{\lambda_{v}}{\sqrt{\lambda_{u}^{2}+\lambda_{v}^{2}}}
$$

As a result, the optimal thrust vector direction provided by the SEP system coincides with that of the primer vector [8] whose radial and transverse components are $\lambda_{u}$ and $\lambda_{v}$, respectively. The optimal control law for $\tau$ is found by observing that $H^{\prime}$ depends linearly on $\tau$. As a result, a bang-bang control is optimal [9]

$$
\tau= \begin{cases}1 & \text { if } \frac{\sqrt{\lambda_{u}^{2}+\lambda_{v}^{2}}}{m}-\frac{\lambda_{m}}{v_{e}}>0 \\ 0 & \text { if } \frac{\sqrt{\lambda_{u}^{2}+\lambda_{v}^{2}}}{m}-\frac{\lambda_{m}}{v_{e}} \leq 0\end{cases}
$$

A similar situation occurs with $\eta$, whose optimal control law is simply given by

$$
\eta= \begin{cases}\eta_{\max } & \text { if } \lambda_{u}>0 \\ 0 & \text { if } \lambda_{u} \leq 0\end{cases}
$$

\section{Numerical Simulations}

A set of canonical units [11] have been used in the integration of the differential equations to reduce their numerical sensitivity. The differential Eqs. (4-8) and (12-15) have been integrated in double precision using a Runge-Kutta-fifth-order scheme with absolute and relative errors of $10^{-12}$. The boundary value problem associated with the variational problem has been solved by means of a hybrid technique that combines gradient-based and direct methods [12].

The control laws described earlier have been applied to study the rendezvous trajectories towards Venus $\left(r_{f}=r_{\varphi}=0.72333199 \mathrm{AU}\right)$ and Mars $\left(r_{f}=r_{\lambda}=1.52366231 \mathrm{AU}\right)$ for values of $\eta_{\max }$ ranging in the interval $[0,0.5]$. The characteristics of the SEP system and the launch mass are consistent with that of the European SMART-1 spacecraft, and the corresponding data have been taken from [13]. In 

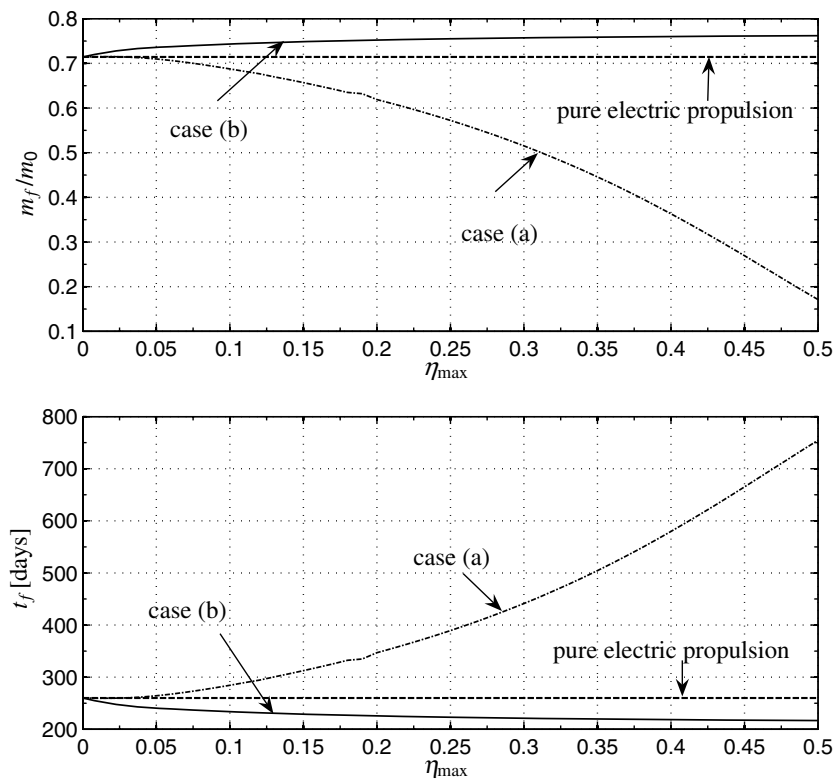

Fig. 3 Results for an Earth-Venus circle-to-circle rendezvous mission.
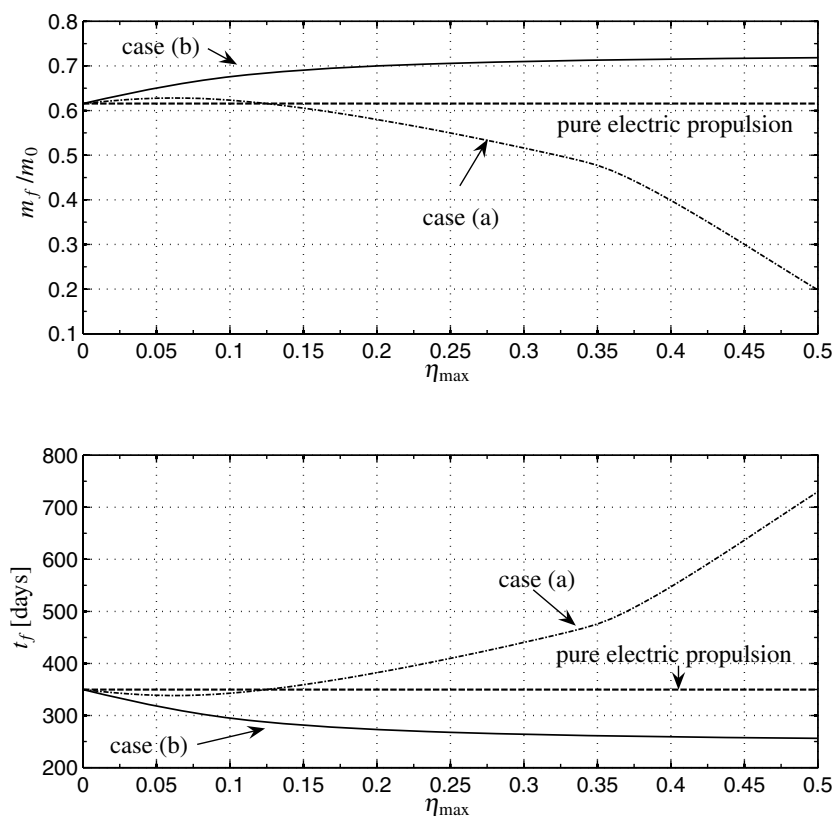

Fig. 4 Results for an Earth-Mars circle-to-circle rendezvous mission.

particular, a SEP thrust $T_{e}=73 \mathrm{mN}$, an exhaust velocity $v_{e}=16.4 \mathrm{~km} / \mathrm{s}$, and an initial spacecraft mass $m_{0}=350 \mathrm{~kg}$ have been assumed.

To better highlight the role of the auxiliary system on the mass necessary to accomplish the mission, the following two cases have been considered.

Case a: The parameter $\eta$ is held fixed for the whole trajectory using a value coincident with its maximum attainable value $\eta \equiv \eta_{\max }=$ const. This case is representative of a situation in which $T_{a}$ cannot be modulated nor zeroed. The optimal control law for the other two control variables $\tau$ and $\alpha$ is given by Eqs. (21) and (22).

Case b: the parameter $\eta \in\left[0, \eta_{\max }\right]$ is actually a control variable and its steering law is provided by Eq. (23).

Figures 3 and 4 show the simulation results, for the two rendezvous test missions, as a function of different values of $\eta_{\max }$. To better quantify the impact of the auxiliary system over the mission performance, the two figures also display the case corresponding to the employment of a SEP thruster with no auxiliary system $\left(T_{a}=0\right)$ during the whole mission.

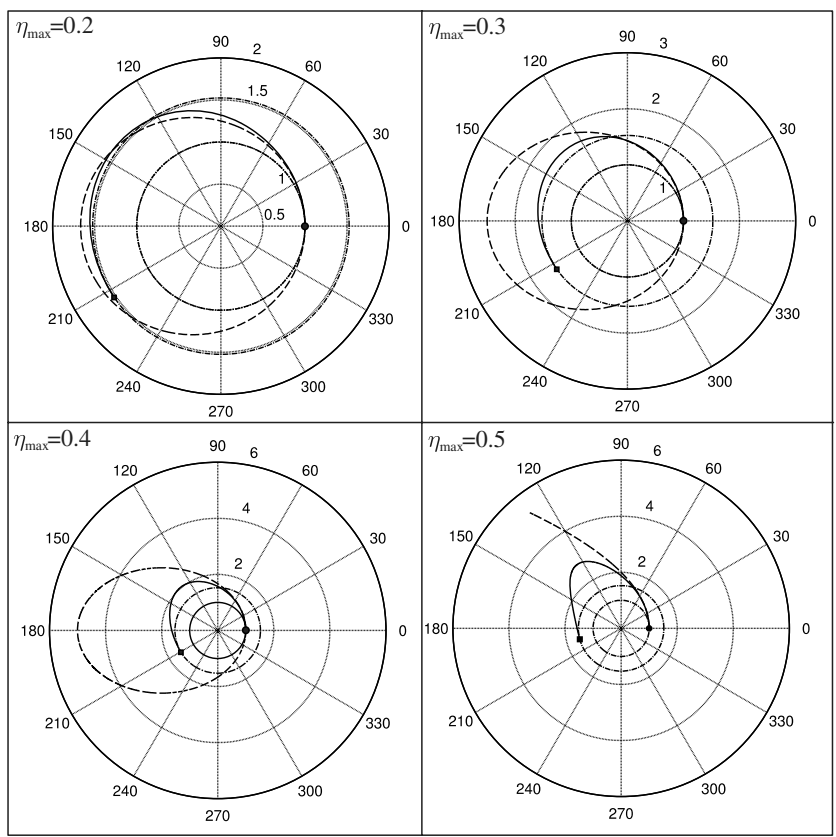

Fig. 5 Earth-Mars trajectories inclusive of the start (circle) and arrival (square) spacecraft positions.

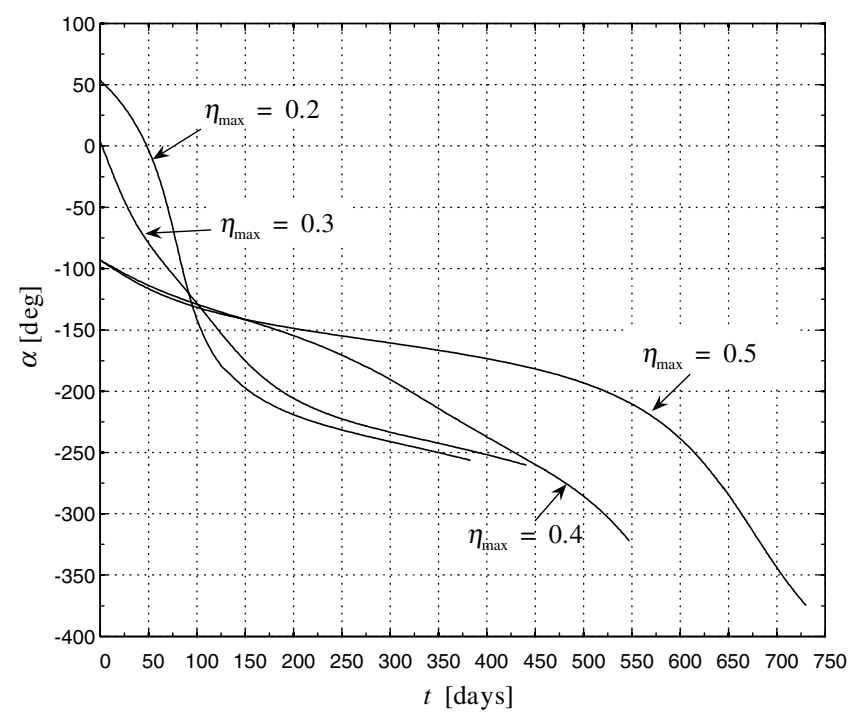

Fig. 6 Time histories of $\alpha$ for an Earth-Mars transfer (case a).

As expected, the performance in terms of final mass and mission time are much worse in case a than in case b (in fact, the latter coincides with the optimal condition). There are other points worth noting. First, the performance in case a is also worse (except for small values of $\eta_{\max }$ ) than that corresponding to a pure SEP thruster. Moreover, the performance in case a tends to degrade quickly as $\eta_{\max }$ is increased. This is a counterintuitive behavior because one might expect that a decrease in the solar gravitational attraction (as happens when the auxiliary system is turned on) could increase the effectiveness of the SEP system, at least as long as missions towards outer planets are considered. However, this is a wrong conclusion. In fact, the presence of a significant and constant value of $\eta$ forces the spacecraft into an initial highly elliptic trajectory [6], setting in this way the aphelion well beyond the radius of the target orbit, see Fig. 5 (continuous lines represent case a). As a result, the electric thruster in the initial mission phase is not used to accelerate but rather to decelerate the spacecraft, so as to counteract the negative effect of the auxiliary system. This characteristic is clearly seen in Fig. 6 in which the time histories of $\alpha$, corresponding to case a, are shown for an 

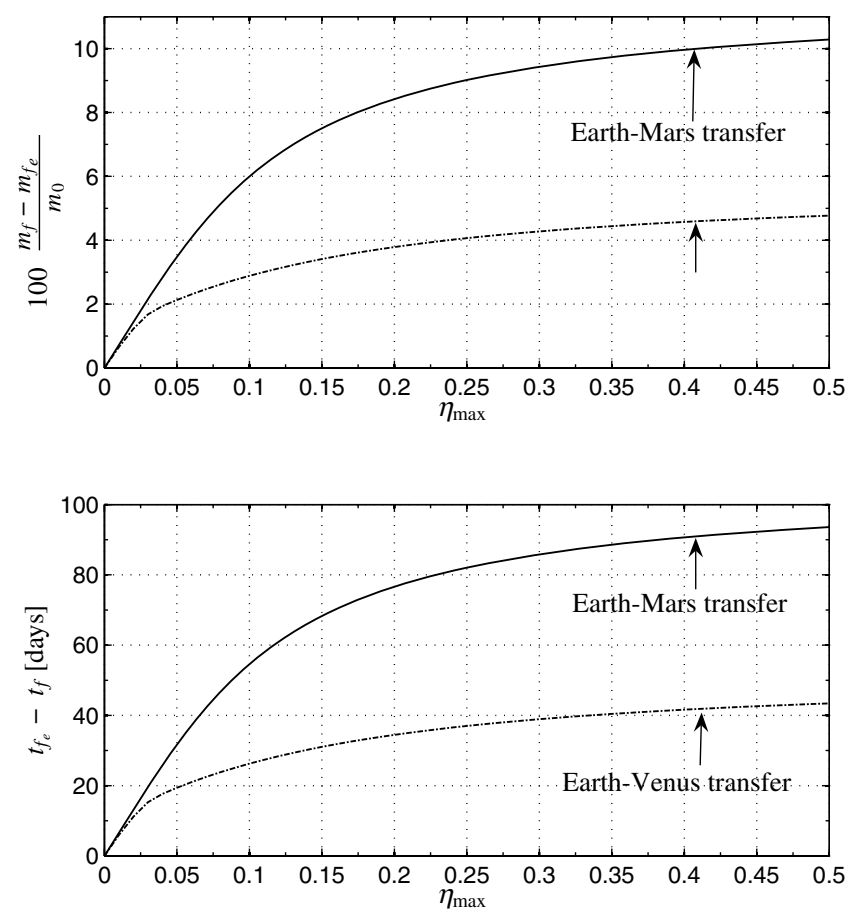

Fig. 7 Mass and mission time savings for case $b$ with respect to a pure SEP mission.

Earth-Mars mission using four different values of $\eta_{\max }$. For example, when $\eta_{\max }=0.4$ and $\eta_{\max }=0.5$, the electric thruster direction angle is $\alpha=-100 \mathrm{deg}$ in the initial mission phase. Figure 5 also shows the ballistic trajectories (dotted lines) that the spacecraft would follow without the engagement of the SEP system. Note that when $\eta_{\max }=0.5$, the trajectory turns into a parabola, that is an escape trajectory. In fact, from the conservation of energy, the ratio of the spacecraft escape velocity $v_{\text {esc }}$ at instant $t_{0}$ to the initial circular velocity $v_{c}=\sqrt{\mu_{\odot} / r_{0}}$ is found to be

$$
\frac{v_{\mathrm{esc}}}{\sqrt{\mu_{\odot} / r_{0}}}=\sqrt{2\left(1-\eta_{\max }\right)}
$$

Equation (24) shows that when $\eta_{\max }=0.5, v_{\text {esc }} / v_{c}=1$, which corresponds to attaining an escape condition.

Another interesting point emerging from Figs. 3 and 4 is that case $b$ offers, for Mars and Venus missions and for any values of $\eta_{\max }$, a better performance than that obtainable from a pure SEP system, both in terms of propellant consumption and mission time. The mass and the transfer time saving are shown in Fig. 7 where the terms $m_{f_{e}}$ and $t_{f_{e}}$ refer to the final mass and flight time of a pure SEP based mission $\left(\eta_{\max }=0\right)$.

The mission trajectory and the time history of the control angle $\alpha$ for case $\mathrm{b}$ are shown in Fig. 8 for $\eta_{\max }=0$ and $\eta_{\max }=0.5$. We note that the auxiliary system is only engaged in the early transfer phase (thick line of Fig. 8b) and that a similar behavior is found in all of the analyzed trajectories. Observe that, as long as the auxiliary system is operating, $\eta=\eta_{\max }$, as implied by Eq. (23).

Figure 7 shows that both the propellant mass and the flight-time saving are increasing functions of $\eta_{\max }$. In particular, when $\eta_{\max }=$ 0.5 the mass saving is about 10 and $5 \%$ for Earth-Mars and EarthVenus missions while the corresponding time saving is 95 and 45 days, respectively. Figure 7 also shows a steep growth of mass and time savings for small values of $\eta_{\max }$, whereas the curve slopes tend to quickly decrease as $\eta_{\max }$ is increased. This behavior implies that it would not be a good idea to choose auxiliary systems with high values of $\eta_{\max }$. Anyway, the fundamental information about the effectiveness of a HLTP system over a pure SEP system is ultimately
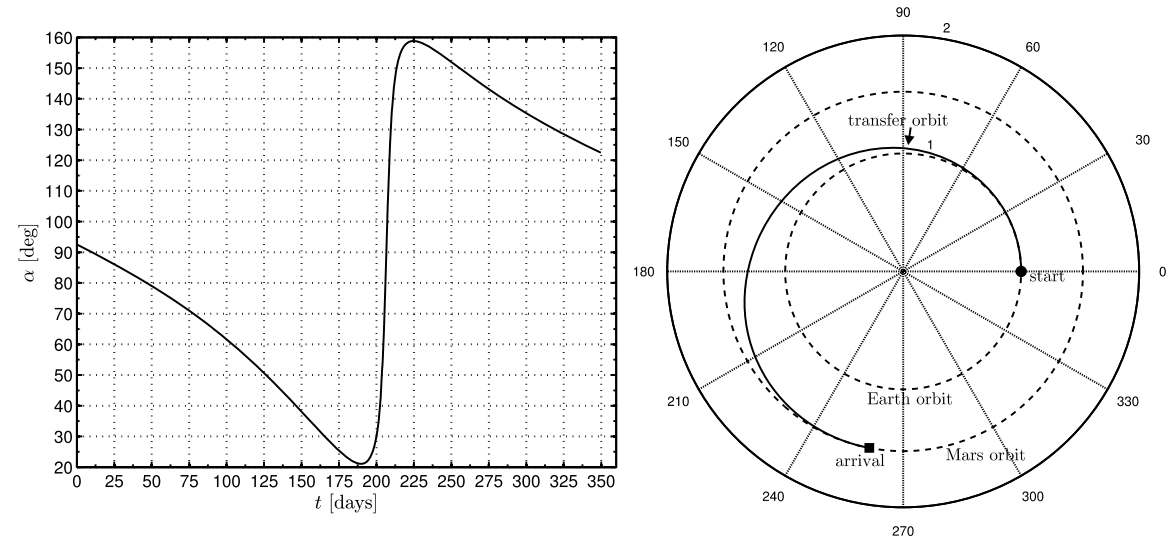

a) Pure electric propulsion $\left(\eta_{\max }=0\right)$
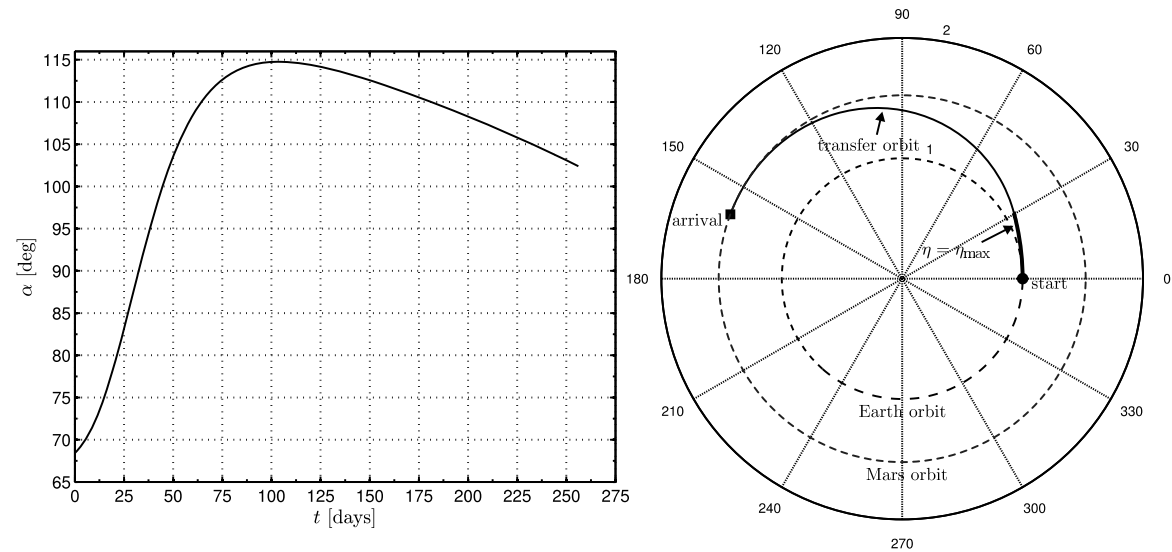

b) Case (b) $\left(\eta_{\max }=\mathbf{0 . 5}\right)$

Fig. 8 Earth-Mars trajectories and time histories of the control angle $\alpha$. 
given in terms of payload mass deliverable for a given spacecraft mass. This subject is addressed in the next section.

\section{Payload Mass Fraction}

So far, we have quantified the advantages of an auxiliary system in terms of $\eta_{\max }$, a parameter useful to define the performance of the system. However, the additional thrust system increases the spacecraft mass so that the question arises whether there exists a benefit in terms of payload mass fraction deliverable. We need to find a relationship between the value of $\eta_{\max }$ and the mass of the auxiliary system. To this end, we assume the following mass breakdown model for the spacecraft

$$
m_{0}=m_{e}+m_{\text {prop }}+m_{a}+m_{\text {pay }}
$$

where $m_{e}$ is the mass of the SEP system, $m_{\text {prop }}=m_{\text {prop }}\left(\eta_{\max }\right)$ is the propellant mass (related to the SEP system) necessary to accomplish the mission, $m_{a}=m_{a}\left(\eta_{\max }\right)$ is the mass of the auxiliary system, and $m_{\text {pay }}$ is the sum of the payload mass and the mass of the remaining subsystems.

For a given SEP system (that is, $m_{e}$ is fixed) and for a given value of the initial spacecraft mass $m_{0}$, the impact of the auxiliary propulsive system on the value of $m_{\text {pay }}$ can be estimated through the function $f_{m}=f_{m}\left(\eta_{\max }\right)$ defined as [see Eq. (25)]

$$
f_{m}\left(\eta_{\max }\right) \triangleq \frac{m_{\text {pay }}+m_{e}}{m_{0}}=1-\frac{m_{\text {prop }}\left(\eta_{\max }\right)}{m_{0}}-\frac{m_{a}\left(\eta_{\max }\right)}{m_{0}}
$$

Clearly, $f_{m}(0)$ represents the case in which a pure SEP system is available. Equation (26) allows one to quantify the relative "weight" of the auxiliary system, once a reasonable expression for the mass fraction $m_{a} / m_{0}$ is found. This can be done by introducing the type of auxiliary system. Although, in principle, different systems may be found capable of generating a modulated radial thrust (variable according to an inverse square law with the distance from the sun) in the following, we concentrate on solar sail-based applications.

\section{Solar Sail Application}

Assume that the auxiliary propulsive system is constituted by a solar sail whose effective surface $A$ is kept constant. The solar sail is capable of generating a pure radial thrust provided its reflector is kept perpendicular to the sunlight direction. Of course, the sail may be employed more efficiently by using its capability of supplying a tangential thrust component if tilted away from the radial direction. Nevertheless here we assume a pure radial thrust to be consistent with the previously discussed model. Also, it should be noted that the possible interference of the SEP exhaust plume with the reflector may vary the optical characteristics of the sail. This complex effect is neglected for the sake of simplicity.

We make use of the so-called nonperfect reflection model [14], which takes into account the optical coefficients of the real sail film. The thrust $T_{a}$ provided by a flat sail whose surface is perpendicular to the sun-spacecraft line is given by

$$
T_{a}=\tau_{a} m \Psi \beta_{\sigma} \frac{\mu_{\odot}}{r^{2}}
$$

where $\beta_{\sigma}$ is referred to as dimensionless sail loading, similar to that introduced in [14], $\tau_{a}=(0,1)$ is the solar sail switching parameter, and $\Psi \leq 1$ is a positive coefficient depending on the optical characteristics of the sail film $(\Psi=1$ in the ideal case of specular reflection). For example, the optical coefficient for a sail with a highly reflective aluminum-coated front side and a highly emissive chromium-coated back side is $\Psi=0.90815$ [15]. The dimensionless sail loading is defined as $\beta_{\sigma} \triangleq \sigma^{*} / \sigma$, where $\sigma^{*} \triangleq 1.539 \mathrm{~g} / \mathrm{m}^{2}$ is the critical solar sail loading parameter (see [16], p. 40) and $\sigma \triangleq m / A$ is a generalized sail loading. In fact, $\sigma$ is defined as a function of the whole spacecraft mass and not of the sail mass alone (as is usually done): as such, $\sigma$ is not constant with time. Note that

$$
\beta_{\sigma}=\beta_{\sigma_{0}} \frac{m_{0}}{m}
$$

where

$$
\beta_{\sigma_{0}} \triangleq \sigma^{*} /\left(m_{0} / A\right)
$$

is the initial (constant) dimensionless sail loading. It represents a technological parameter that quantifies the limits of the current technology.

In Eq. (27), the solar sail switching parameter $\tau_{a}$ models the possibility of zeroing the sail thrust by orienting the sail area parallel to the incident solar rays, thus obtaining $\eta=0$. Accordingly, $\tau_{a}$ may be thought of as a sort of solar sail activation parameter. The presence of $\tau_{a}$ in the mathematical model guarantees the possibility of realizing an on/off control over $\eta$ (recall that $A$ is assumed to be constant). On the other hand, the feasibility of a continuous variation of $\eta$ between suitable limits by changing the effective sail area (as suggested in [6]) appears to be of difficult practical implementation.

Substituting Eq. (28) into Eq. (27) and comparing the latter with Eq. (3), the following expression for $\eta$ is found:

$$
\eta=\tau_{a} \Psi \beta_{\sigma_{0}} \frac{m_{0}}{m}=\tau_{a} \tilde{\beta}_{\sigma_{0}} \frac{m_{0}}{m}
$$

where $\tilde{\beta}_{\sigma_{0}} \triangleq \Psi \beta_{\sigma_{0}}$ is the initial dimensionless sail loading, weighted by the coefficient $\Psi$. Equation (30) states that $\eta$ depends on the switching parameter $\tau_{a}$, on the constant coefficient $\tilde{\beta}_{\sigma_{0}}$ (that defines the maximum performance of the auxiliary system), and on the instantaneous mass ratio through the term $m_{0} / m$. In particular, the presence of the instantaneous mass ratio into the expression of $\eta$ renders the optimal control problem substantially different from that previously analyzed. In fact, although both the equations of motion (4-8) and the Hamiltonian (10) are still formally valid, when Eq. (30) is substituted into the Hamiltonian, the new control vector $\boldsymbol{u} \triangleq$ $\left[\tau_{a}, \tau, \alpha\right]$ now must be optimized. It is a simple matter to verify that the Euler-Lagrange Eqs. (11-14) remain unchanged, whereas the differential equation involving the mass costate $\lambda_{m}$ becomes

$$
\dot{\lambda}_{m}=\frac{\tau T_{e}}{m^{2}}\left(\lambda_{u} \cos \alpha+\lambda_{v} \sin \alpha\right)+\tau_{a} \lambda_{u} \tilde{\beta}_{\sigma_{0}} \frac{\mu_{\odot}}{r^{2}} \frac{m_{0}}{m^{2}}
$$

Equation (31) replaces Eq. (15). As a result, both the optimal control law for the electric thruster direction angle $\alpha$ and for the electric thruster switching parameter $\tau$ are still given by Eqs. (21) and (22), respectively. On the other hand, because the Hamiltonian is linear with respect to $\tau_{a}$, the following bang-bang control law is obtained for the solar sail switching parameter

$$
\tau_{a}= \begin{cases}1 & \text { if } \lambda_{u}>0 \\ 0 & \text { if } \lambda_{u} \leq 0\end{cases}
$$

The performance of a HLTP system, using a solar sail as the auxiliary propulsion system, has been investigated for Earth-Mars and EarthVenus rendezvous missions. The differences with respect to a pure SEP system, in terms of percentage mass ratio and mission time savings, are shown in Fig. 9 as a function of the parameter $\tilde{\beta}_{\sigma_{0}}$. Comparing the results of Fig. 9 with those obtained in the previous section and summarized in Fig. 7, one discovers an almost exact equivalence between the two figures (when $\eta_{\max }$ and $\tilde{\beta}_{\sigma_{0}}$ are interchanged). This is an interesting circumstance as the results refer to two distinct optimal control problems.

The explanation of such a behavior comes from the analysis of the time history of the control variable $\tau_{a}$. In fact, for all the values of $\tilde{\beta}_{\sigma_{0}}$ in the range $[0,0.5], \tau_{a}$ happens to be different from zero in the early mission phase only. This amounts to saying that the auxiliary propulsive system is operating for a fraction of the whole mission time (on the order of 10-15\%) when the spacecraft mass is about its initial value $m_{0}$. Recalling the optimal control law for $\eta$ [see Eq. (23)] and bearing in mind that $\eta$, similarly to $\tau_{a}$, is different from zero in the first mission phase only (see, for example, Fig. 8b), one has 

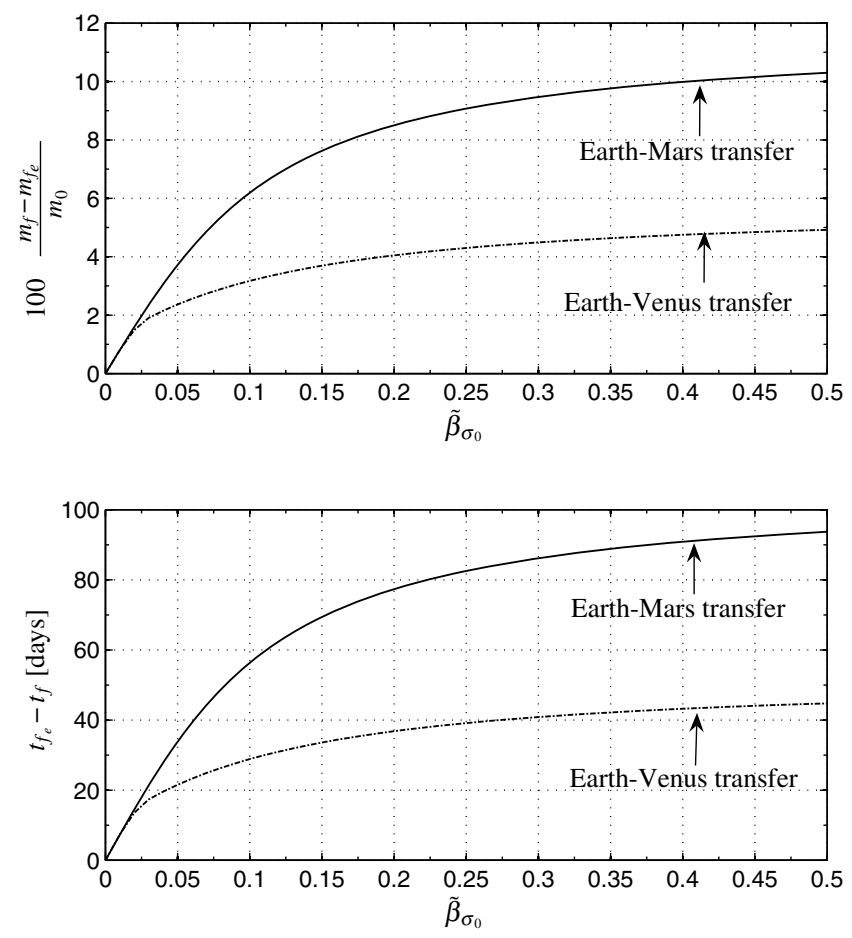

Fig. 9 Mass and mission time savings for a HLTP system with solar sail with respect to a pure SEP mission.
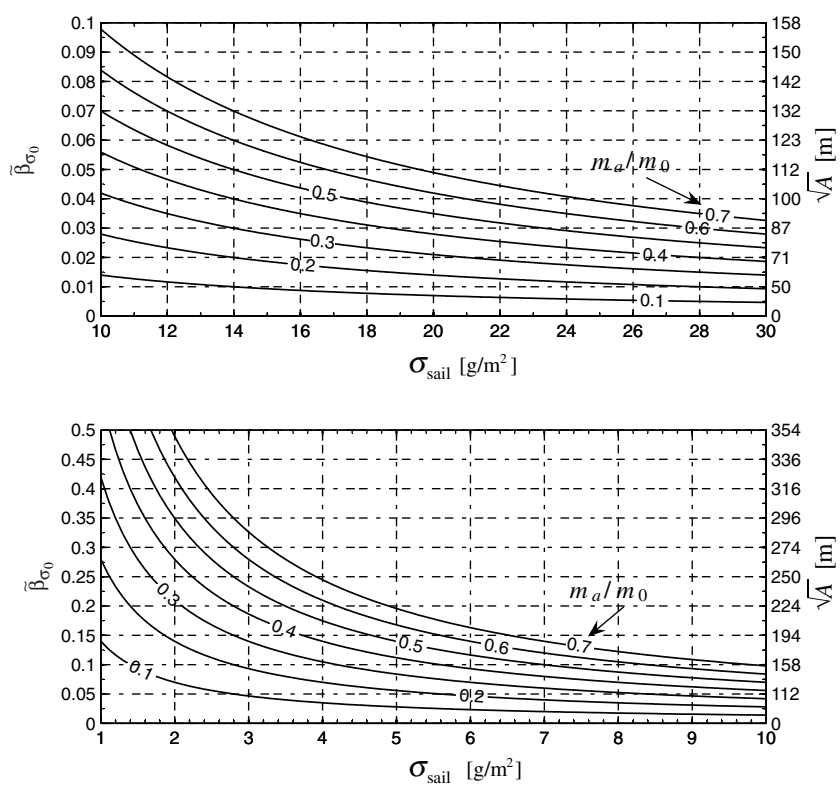

Fig. 10 Dependence of $\tilde{\beta}_{\sigma_{0}}$ and of $\sqrt{A}$ on $\sigma_{\text {sail }}$ as a function of the mass fraction.

$$
\eta \neq 0 \Rightarrow \eta=\eta_{\max }, \quad \tau_{a} \neq 0 \Rightarrow \tau_{a}=1
$$

so that from Eq. (30) one concludes that $\eta_{\max }=\tilde{\beta}_{\sigma_{0}}\left(m / m_{0}\right) \cong \tilde{\beta}_{\sigma_{0}}$. In other terms, it turns out that there is a substantial equivalence between $\eta_{\max }$ and $\tilde{\beta}_{\sigma_{0}}$, and Fig. 7 can be used to quantify the performance of a HLTP system with a solar sail as the auxiliary system.

Having expressed the mass fraction $m_{f} / m_{0}$ as a function of the design parameter $\tilde{\beta}_{\sigma_{0}}$ (or, equivalently, as a function of $\eta_{\max }$ ), it is now possible to study the function $f_{m}$ defined through Eq. (26). To this end it is necessary to establish a suitable expression for the mass fraction $m_{a} / m_{0}$ associated with the auxiliary system, that is, with the
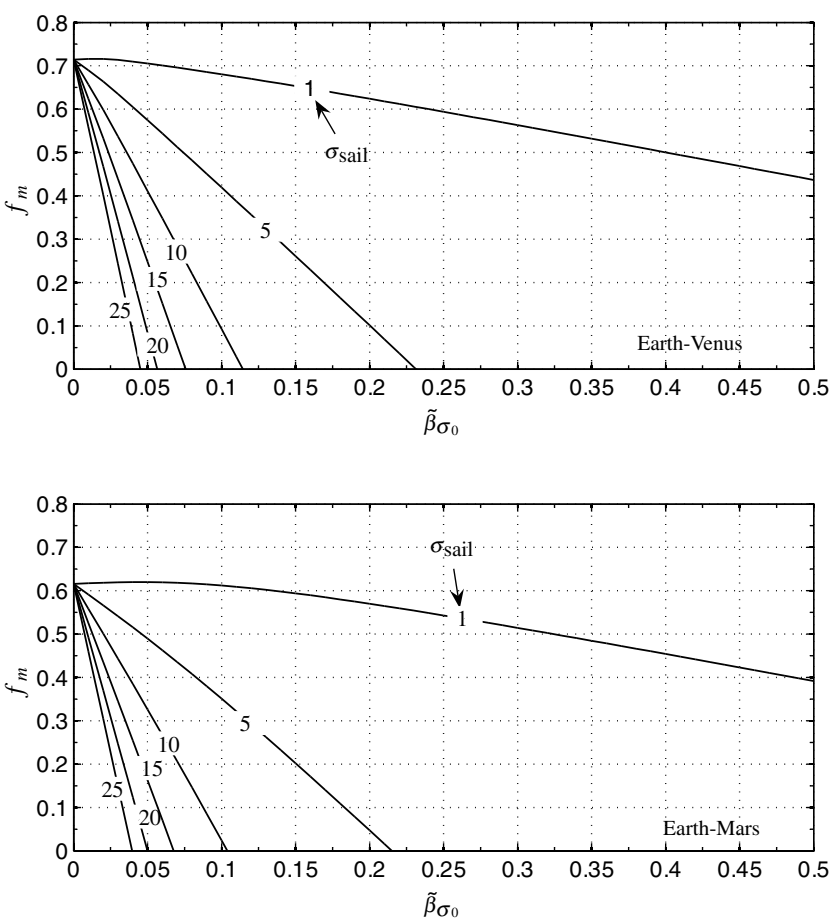

Fig. 11 Dependence of $f_{m}$ [see Eq. (35)] on $\tilde{\beta}_{\sigma_{0}}$ for some values of $\sigma_{\text {sail }}$ $\left(\mathrm{g} / \mathrm{m}^{2}\right)$.

solar sail. First, introduce the sail assembly loading $[17,18]$ $\sigma_{\text {sail }} \triangleq m_{a} / A$, i.e., the mass of the sail assembly (comprising the sail film and the required structure for storing, deploying and tensioning the sail) per unit area. Then, Eq. (29) yields

$$
\frac{m_{a}}{m_{0}}=\frac{\sigma_{\text {sail }}}{\sigma^{*}} \beta_{\sigma_{0}}=\frac{\sigma_{\text {sail }}}{\sigma^{*}} \frac{\tilde{\beta}_{\sigma_{0}}}{\Psi}
$$

The value of $\sigma_{\text {sail }}$ depends on the technology employed to build the sail and, in particular, is closely related to the value of the density of the sail film. Currently, admissible values are on the order of $25-30 \mathrm{~g} / \mathrm{m}^{2}$, even if near-term and midterm technology $[18,19]$ will hopefully allow values of $5-10 \mathrm{~g} / \mathrm{m}^{2}$. However, future outer solar system missions will require an assembly loading of the order of $1 \mathrm{~g} / \mathrm{m}^{2}$, see [16], p. 95. Figure 10 shows the dependence of $\tilde{\beta}_{\sigma_{0}}$ on the assembly loading $\sigma_{\text {sail }}$ as a function of the mass fraction $m_{a} / m_{0}$. The figure also shows the corresponding value of the sail characteristic dimension $(\sqrt{A})$. The latter coincides with the side length for a square sail configuration. Assuming, for example, an assembly loading of $25 \mathrm{~g} / \mathrm{m}^{2}$ and a mass fraction equal to 0.5 , Fig. 10 shows that $\tilde{\beta}_{\sigma_{0}} \cong 0.028$ (recall that this value is approximately equal to $\left.\eta_{\max }\right)$.

Bearing in mind Eqs. (26) and (34) one has

$$
f_{m}=\frac{m_{f}}{m_{0}}-\frac{\sigma_{\text {sail }}}{\sigma^{*}} \frac{\tilde{\beta}_{\sigma_{0}}}{\Psi}
$$

The function $f_{m}$ has been drawn in Fig. 11 for some values of the sail loading. Figure 11 shows that, with the exception of very highperformance solar sails, function $f_{m}$ is always decreasing with respect to $\tilde{\beta}_{\sigma_{0}}$. More precisely, $\partial f_{m} / \partial \tilde{\beta}_{\sigma_{0}}<0$ if $\sigma_{\text {sail }}>2 \mathrm{~g} / \mathrm{m}^{2}$ for both the analyzed missions. These results indicate that, for current and near-midterm technology, the use of a solar sail as an auxiliary propulsive system produces a decrease of the payload mass fraction deliverable. In fact, the extra mass of the solar sail is greater than the propellant mass that is saved with a pure SEP system (recall that the latter is represented by the point $f_{m}(0)$ in Fig. 11). Nevertheless, because an increase of $\tilde{\beta}_{\sigma_{0}}$ makes the mission time decrease (see Fig. 9), a HLTP system can be usefully employed in the mission 
analysis to trade off the conflicting requirements between deliverable payload mass fraction and mission time.

\section{Conclusions}

A novel concept of mission, based on a hybrid low-thrust propulsion system, has been proposed and discussed. A solar electric propulsion thruster has been coupled with an auxiliary system, capable of providing an inverse square radial thrust. The idea is that, by virtue of this additional thrust, the spacecraft "senses" a reduced gravitational solar force. As a result, the propellant expense for a given heliocentric mission is typically decreased. To quantify this effect on a rigorous basis the problem has been cast into an optimality framework. In particular, the steering law that minimizes the propellant consumption for a circle-to-circle rendezvous problem has been found using an indirect approach. Exploiting the size of the reduction of the gravitational acceleration as a control parameter, it has been shown that an increase of that parameter provides not only better performance in terms of propellant consumption but also in terms of mission time (which decreases as long as the value of the net gravitational acceleration decreases). To make a meaningful comparison between a hybrid and a conventional electric propulsion system, these two options have been compared in terms of payload mass fraction deliverable for a given mission. To illustrate such a comparison, a solar sail has been used as an auxiliary system. It has been shown that the advantage of a hybrid system is subordinated to the employment of high-performance solar sails. Nevertheless, the reduction in mission times (whatever the solar sail characteristics be) of the new hybrid system makes this configuration attractive for future planetary missions. Other types of low-thrust hybridization are possible, such as solar electric power and minimagnetospheric plasma propulsion.

\section{References}

[1] Williams, S. N., and Coverstone-Carroll, V., "Benefits of Solar Electric Propulsion for the Next Generation of Planetary Exploration Missions," Journal of the Astronautical Sciences, Vol. 45, No. 2, April-June 1997, pp. 143-159.

[2] Williams, S. N., and Coverstone-Carroll, V., "Mars Missions Using Solar Electric Propulsion," Journal of Spacecraft and Rockets, Vol. 37, No. 1, Jan.-Feb. 2000, pp. 71-77.

[3] Brophy, J. R., "Advanced Ion Propulsion Systems for Affordable DeepSpace Missions," Acta Astronautica, Vol. 52, Nos. 2-6, Jan.March 2003, pp. 309-316.
[4] Dachwald, B., "Optimization of Interplanetary Solar Sailcraft Trajectories Using Evolutionary Neurocontrol," Journal of Guidance, Control, and Dynamics, Vol. 27, No. 1, 2004, pp. 66-72.

[5] Zubrin, R. M., and Andrews, D. G., "Magnetic Sails and Interplanetary Travel," Journal of Spacecraft and Rockets, Vol. 22, No. 2, 1991, pp. 197-203.

[6] McInnes, C. R., "Orbits in a Generalized Two-Body Problem," Journal of Guidance, Control, and Dynamics, Vol. 26, No. 5, Sept.-Oct. 2003, pp. 743-749.

[7] Leipold, M., and Götz, M., "Hybrid Photonic/Electric Propulsion," Kayser-Threde, GmBH, Rept. SOL4- TR-KTH-0001, Munich, Germany, Jan. 2002.

[8] Lawden, D. F., Optimal Trajectories for Space Navigation, Butterworths, London, 1963, pp. 54-68.

[9] Prussing, J. E., and Chiu, J. H., "Optimal Multiple-Impulse Time-Fixed Rendezvous Between Circular Orbits," AIAA Paper 1984-2036, Aug. 1984.

[10] Bryson, A. E., and Ho, Y. C., Applied Optimal Control, Hemisphere, New York, 1975, Chap. 2, pp. 71-86.

[11] Seidelmann, K. P., Explanatory Supplement to the Astronomical Almanac, University Science Books, Mill Valley, CA, 1992, pp. 696697.

[12] Mengali, G., and Quarta, A. A., "Optimal Three-Dimensional Interplanetary Rendezvous Using Nonideal Solar Sail," Journal of Guidance, Control, and Dynamics, Vol. 28, No. 1, Jan.-Feb. 2005, pp. 173-177.

[13] Schoenmaekers, J., "Post-Launch Optimization of the SMART-1 LowThrust Trajectory to the Moon," Proceedings of the 18th International Symposium on Space Flight Dynamics, SP-548, ESA, 2004.

[14] Dachwald, B., Mengali, G., Quarta, A. A., and Macdonald, M., "Parametric Model and Optimal Control of Solar Sails with Optical Degradation," Journal of Guidance, Control, and Dynamics (to be published).

[15] Wright, J. L., Space Sailing, Gordon and Breach, Philadelphia, 1992, pp. 227-233.

[16] McInnes, C. R., Solar Sailing: Technology, Dynamics and Mission Applications, Springer-Praxis Series in Space Science and Technology, Springer-Verlag, Berlin, 1999, pp. 40, 95.

[17] McInnes, C. R., "Payload Mass Fractions for MinimumTime Trajectories of Flat and Compound Solar Sails," Journal of Guidance, Control, and Dynamics, Vol. 23, No. 6, 2000, pp. 10761078.

[18] Dachwald, B., "Solar Sail Performance Requirements for Missions to the Outer Solar System and Beyond," Proceedings of the 55th International Astronautical Congress, International Astronautical Congress Paper IAC-04-S.P.11, Oct. 2004.

[19] Gershman, R., and Seybold, C., "Propulsion Trades for Space Science Missions," Acta Astronautica, Vol. 45, Nos. 4-9, 1999, pp. 541-548. 